\title{
Betaine reduces $\beta$-amyloid-induced paralysis through activation of cystathionine- $\beta$-synthase in an Alzheimer model of Caenorhabditis elegans
}

\author{
Anne Leiteritz, Benjamin Dilberger, Uwe Wenzel and Elena Fitzenberger ${ }^{*}$ (D)
}

\begin{abstract}
Background: The neurodegenerative disorder Alzheimer's disease is caused by the accumulation of toxic aggregates of $\beta$-amyloid in the human brain. On the one hand, hyperhomocysteinemia has been shown to be a risk factor for cognitive decline in Alzheimer's disease. On the other hand, betaine has been demonstrated to attenuate Alzheimerlike pathological changes induced by homocysteine. It is reasonable to conclude that this is due to triggering the remethylation pathway mediated by betaine-homocysteine-methyltransferase. In the present study, we used the transgenic Caenorhabditis elegans strain CL2006, to test whether betaine is able to reduce $\beta$-amyloid-induced paralysis in C. elegans. This model expresses human $\beta$-amyloid 1-42 under control of a muscle-specific promoter that leads to progressive, age-dependent paralysis in the nematodes.
\end{abstract}

Results: Betaine at a concentration of $100 \mu \mathrm{M}$ was able to reduce homocysteine levels in the presence and absence of $1 \mathrm{mM}$ homocysteine. Simultaneously, betaine both reduced normal paralysis rates in the absence of homocysteine and increased paralysis rates triggered by addition of homocysteine. Knockdown of cystathionine- $\beta$-synthase using RNA interference both increased homocysteine levels and paralysis. Additionally, it prevented the reducing effects of betaine on homocysteine levels and paralysis.

Conclusion: Our studies show that betaine is able to reduce homocysteine levels and $\beta$-amyloid-induced toxicity in a $C$. elegans model for Alzheimer's disease. This effect is independent of the remethylation pathway but requires the transsulfuration pathway mediated by cystathionine- $\beta$-synthase.

Keywords: Alzheimer's disease, $\beta$-Amyloid, Caenorhabditis elegans, Hyperhomocysteinemia, Betaine

\section{Background}

Alzheimer's disease (AD) is the most common agerelated neurodegenerative disorder caused by the accumulation of aggregated $\beta$-amyloid $(A \beta)$ as senile plaques in the human brain $[1,2]$. These deposits are associated with synaptotoxicity that leads to the characteristic cognitive decline [3]. Moderately elevated plasma homocysteine (Hcy) levels were identified as a strong risk factor not only for vascular dementia but also for AD [4]. Several mechanisms underlying the noxious

\footnotetext{
* Correspondence: elena.fitzenberger@ernaehrung.uni-giessen.de Molecular Nutrition Research, Interdisciplinary Research Center, Justus-Liebig-University of Giessen, Heinrich-Buff-Ring 26-32, 35392 Giessen, Germany
}

(c) The Author(s). 2018 Open Access This article is distributed under the terms of the Creative Commons Attribution 4.0 International License (http://creativecommons.org/licenses/by/4.0/), which permits unrestricted use, distribution, and reproduction in any medium, provided you give appropriate credit to the original author(s) and the source, provide a link to the Creative Commons license, and indicate if changes were made. The Creative Commons Public Domain Dedication waiver (http://creativecommons.org/publicdomain/zero/1.0/) applies to the data made available in this article, unless otherwise stated. effect of Hcy in the brain have been proposed. These include DNA damage [5], activation of $N$-methyl-D-aspartate receptors [6], and the alteration of the amyloid precursor protein (APP) metabolic pathway by hypomethylation [7]. Slightly increased homocysteine levels can be due to single nucleotide polymorphisms in the gene encoding 5,10-methylene-tetrahydrofolate-reductase (MTHFR) [8]. It has been shown that in such cases folic acid supplementation reduces Hcy levels [9]. Alternatively, remethylation of Hcy to methionine and S-adenosylmethionine (SAM) can also be achieved by supplementing betaine as a methyl donor [10]. Indeed, in hyperhomocysteinemic rats betaine supplementation was shown to ameliorate Hcy-induced AD-like pathological changes and memory deficits [11]. In 
the nematode Caenorhabditis elegans, however, effects of betaine on remethylation of Hcy can be excluded since the nematode has no orthologue of betaine-Hcymethyltransferase (BHMT), encoding the relevant enzyme for betaine mediated remethylation [12]. Another biochemical pathway, through which Hcy can be detoxified, is transsulfuration of Hcy to cystathionine by the vitamin B6-dependent enzyme cystathionin- $\beta$-synthase (CBS).

In the present study, we used the transgenic $C$. elegans strain CL2006, which expresses the human $A \beta$ under the control of the muscle-specific unc-54 promotor and displays progressive paralysis [13], in order to test the effects of betaine supplementation on the AD phenotype. To explore the contribution of CBS on the measured effects, RNA interference was used. Moreover, the effects of betaine intervention and CBS knockdown on Hcy levels were estimated by ELISA.

\section{Methods}

\section{Strains}

The transgenic nematode strain CL2006 ((dvIs2[pCL12 (unc-54/human $\mathrm{A} \beta_{1-42}$ minigene) + pRF4]) was obtained from the $C$. elegans Genetics Center, CGC (University of Minnesota, MN, USA). CL2006 expresses human $A \beta_{1-42}$ under the control of the muscle-specific promoter $u n c-54$, leading to progressive, adult-onset paralysis [13]. E. coli HT115 RNAi clones were purchased from Source Bioscience (Cambridge, UK) and included a negative control (empty L4440 vector), cbs-1 (ZC373.1), gcs-1 (F37B12.2), and metr-1 (R03D7.1).

\section{C. elegans maintenance}

The maintenance and experimental procedures were conducted according to standard protocols. The nematodes were cultivated at $20{ }^{\circ} \mathrm{C}$ on nematode growth medium (NGM) agar plates that contained an E. coli OP50 lawn as the major food source [14]. For the experiments, synchronous populations of larvae were used, which were obtained by bleaching egg-laying adults with a hypochlorite solution [15].

\section{RNA interference}

RNA interference (RNAi) was performed by using the feeding method. As described elsewhere, it was conducted in liquid cultures, which were enriched with RNAi bacteria [16, 17]. In brief, in the RNAse IIIdeficient $E$. coli strain HT115 gene-specific dsRNA expression was induced by incubation with $1 \mathrm{mM}$ isopropyl- $\beta$-D-thiogalactopyranoside for $4 \mathrm{~h}$ at $37{ }^{\circ} \mathrm{C}$. Subsequently, the bacteria were centrifuged and resuspended in NGM, containing $50 \mu \mathrm{g} / \mathrm{ml}$ kanamycin to inactivate bacterial growth. This suspension was applied to each well of a 96-well plate together with a volume of $10 \mu \mathrm{l}$ M9 buffer containing 10-15 synchronized L1 larvae. The volume of the NGM-bacteria-suspension varied between 44 and $46 \mu$, depending on the number of effectors, which were added later. That is, in case of betaine supplementation only, $44 \mu \mathrm{l}$ NGM-bacteria suspension were used, and if Hcy and betaine were applied together, $46 \mu \mathrm{l}$ NGM were added.

\section{Treatment of $C$. elegans with Hcy and betaine}

To young adult CL2006 nematodes, which are characterized by the ability to lay eggs, a volume of $6 \mu$ l betaine or $7 \mu \mathrm{l}$ of both betaine and homocysteine were added. Effector solutions were prepared in M9 buffer and contained tenfold enriched concentrations compared to the final concentration in the experiment. Control nematodes were always treated with the identical volumes of M9 buffer only. Treatments were performed for $48 \mathrm{~h}$ before heat-shock was applied.

\section{Measurement of paralysis}

The expression and aggregation of $A \beta_{1-42}$ in C. elegans results in the paralyzation phenotype, which is described as a movement that is restricted to waving of the head without translocation of the animal [18].

Before scoring paralysis, the nematodes were heatshocked for $1.5 \mathrm{~h}$ at $35{ }^{\circ} \mathrm{C}$ in order to accelerate the paralyzation process and to generate a paralyzation rate in the control population of about $50 \%$. After heat shock, the individual nematodes were transferred with $\mathrm{M} 9$ / Tween $20^{\circ}$ - buffer on NGM agar plates. The paralysis phenotype was examined by visual analysis of 25 nematodes per treatment group. Each nematode was tapped with a bent platinum wire, and subsequently, its moving ability was scored.

\section{Hcy-ELISA}

For determination of Hcy concentrations, a quantitative, competitive human Hcy-ELISA Kit (NeoScientific, Cambridge; USA) was employed. To this end, nematodes were treated with lysis buffer (HEPES $50 \mathrm{mM}, \mathrm{NaCl} 150 \mathrm{mM}$, EDTA $5 \mathrm{mM}$, DTT $2 \mathrm{mM}$ ) and frozen at $-80{ }^{\circ} \mathrm{C}$ to extract proteins. After thawing on ice, worms were homogenized with Peqlab Precellys 24-Dual (VWR, Erlangen, Germany). Subsequently, the concentration of extracted protein was determined by using the Bio-Rad Protein Assay. The measurement of the Hcy concentration was set up according to the instruction manual. In brief, the protein solution and a Hcy-HRP conjugate were added to the wells of the ELISA plate, which were coated with a Hcy-specific antibody. Hcy in the samples competes with the conjugate for binding to the plate-bound antibody. Higher Hcy concentrations in the probes lead to decreased Hcy-HRP conjugate binding. By using a HRP substrate, the amount of captured Hcy-HRP can be quantified colorimetrically. The OD of the probes was compared to a 
standard curve, which was generated by using the included calibration standards.

\section{Calculations and statistics}

For statistical analyses, GraphPad Prism 5.0 software (GraphPad, La Jolla, CA, USA) was used. Analysis of variance (ANOVA) and Bonferroni-Holm multiple comparison test as well as 2-way ANOVA were performed for multiple group comparisons. Differences between two groups were examined with Student's $t$ test. The results shown are representatives of at least three independent experiments and are presented as means \pm SD. Significance levels were assumed as $\left.p<0.05{ }^{(*)}\right), p<0.01{ }^{(* *)}$ ), and $p<0.0011^{(* * *)}$.

\section{Results}

Knockdown of cbs-1 increases Hcy levels and paralysis in CL2006

In order to test whether one-carbon metabolism is functional in C. elegans, we knocked down the genes for $c b s-1$ and and 5-methyltetrahydrofolate-methyltransferase metr1 , which is synonymous for methionine synthase. Knockdown of either gene caused an increase of Hcy levels (Fig. 1a). These results suggest that, with regard to Hcy remethylation and transsulfuration, both enzymes possess the same functional role in C. elegans and in humans.
Moreover, both knockdowns significantly increased the paralysis rate in CL2006 (Fig. 1b).

\section{Hcy levels are directly associated with the paralysis phenotype in CL2006}

Next, we assessed whether the observed paralysis under knockdown of $c b s-1$ and metr-1 are indeed due to the increased Hcy levels. To this end, $100 \mu \mathrm{M}$ betaine was applied in the absence or presence of $1 \mathrm{mM}$ exogenous Hcy. Although the reduction of Hcy levels by betaine treatment was not significant, betaine was able to significantly reduce Hcy levels after application of $1 \mathrm{mM}$ exogenous Hcy (Fig. 2a). Significant interactions of homocysteine and betaine were observed $(\mathrm{p}<0.001)$.Moreover, paralysis rate decreased upon betaine treatment both in the absence of exogenous Hcy and when paralysis was first increased by addition of Hcy to the nematodes (Fig. 2b).

Betaine does no longer affect paralysis under cbs-1 RNAi In order to find out whether the paralysis-reducing effects of betaine depend on the activity of CBS, we knocked down the corresponding gene again. RNAi for $c b s-1$ completely prevented the reducing effect of betaine on Hcy levels (Fig. 3a) and paralysis (Fig. 3b). These findings suggest that, on the one hand, betaine acts via

\section{a}

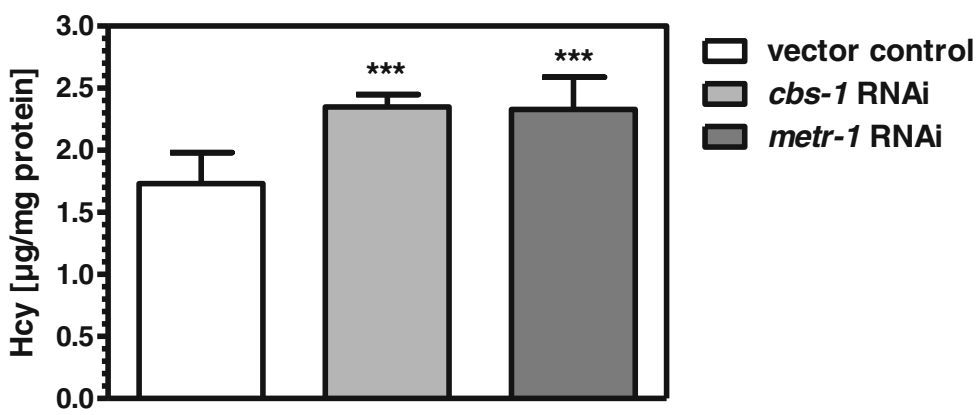

b

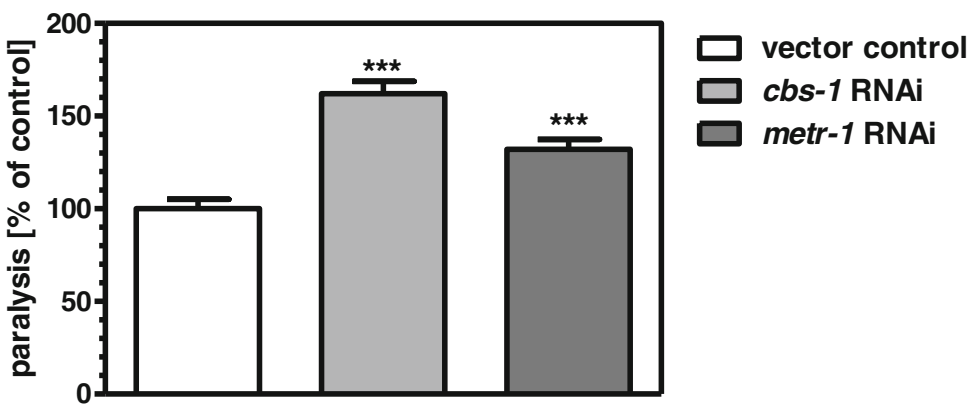

Fig. 1 Knockdown of cbs-1 and metr-1 via RNAi increase Hcy level and paralysis rate in CL2006. Hcy concentration was determined with the Human Hcy-ELISA Kit $48 \mathrm{~h}$ after nematodes had reached the young adult stage (a). At the same, stage paralysis in CL2006 was assessed subsequent to a heatshock for $1.5 \mathrm{~h}$ at $35^{\circ} \mathrm{C}$ by tapping the nematodes with a bent platinum wire and subsequent scoring of their moving ability (b). ${ }^{* *} p<0.001$ versus the vector control 
a

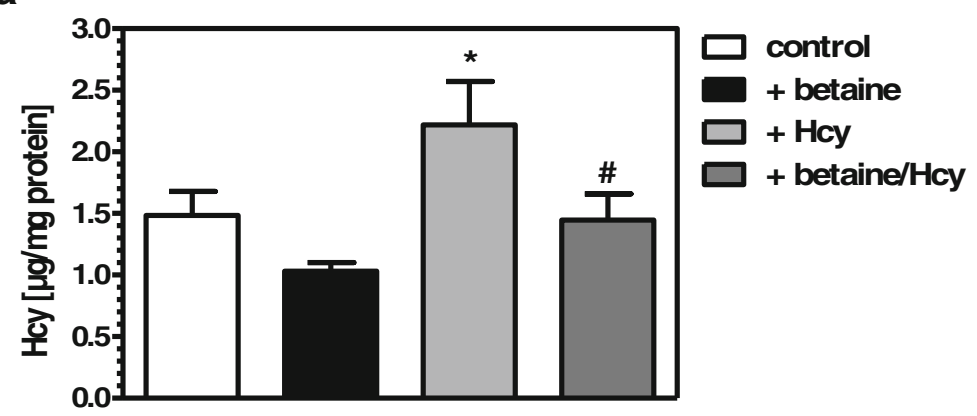

b

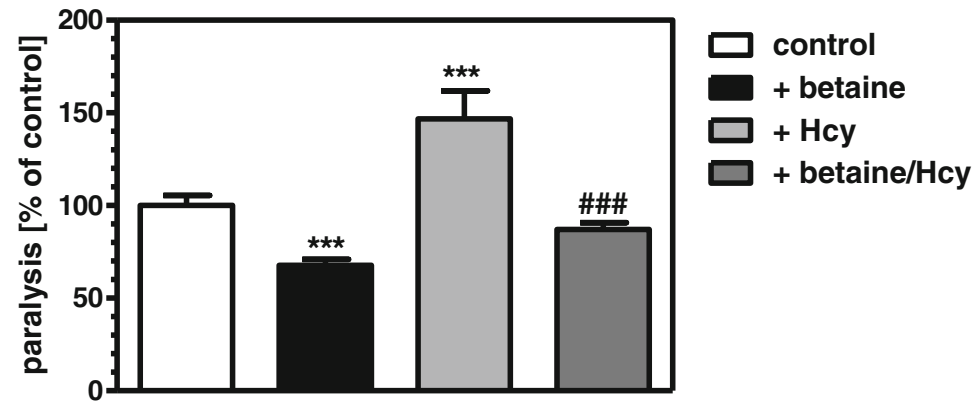

Fig. 2 Betaine reduces Hcy level and paralysis rate in the absence and presence of exogenous Hcy. Hcy level was determined either in control nematodes or those treated with $1 \mathrm{mM} \mathrm{Hcy}$. Moreover, in an additional experiment, both groups were exposed to $100 \mu \mathrm{M}$ betaine also (a). Paralysis was determined under identical conditions as described for Hcy measurements $(\mathbf{b})$. ${ }^{*} p<0.05$, ${ }^{* *} p<0.001$ versus the control; ${ }^{\# \# \#} p<0.001$ versus worms treated with Hcy

triggering the transsulfuration pathway. On the other hand, increased transsulfuration of Hcy would provide more cysteine, which could serve as a substrate for glutathione synthesis. This could be of special importance in preventing the $\mathrm{AD}$-phenotype, since $\mathrm{AD}$ is frequently shown to be associated with increased oxidative stress. However, when we knocked down gcs-1 ( $\gamma$-glutamyl-cysteine-synthetase), encoding the key enzyme for glutathione synthesis, we did not observe any influence on the paralysis rate. Moreover, betaine was still able to reduce paralysis in CL2006 to the same extent as in the presence of GCS-1 (Fig. 3c).

\section{Discussion}

Senile plaques consisting of $\mathrm{A} \beta$ are a hallmark of $\mathrm{AD}$ and are considered as responsible for neuronal damage [19]. It is suggested that $A \beta$ oligomers are neurotoxic because they lead to increased oxidative stress [20], mitochondrial dysfunction [21], disturbed metal ion homeostasis [22], and apoptosis [23]. Hyperhomocysteinemia has been shown to be associated with $\mathrm{AD}$ and represents a factor which is influenced genetically and environmentally [24]. The impact of genetic impairments of one-carbon metabolism, as is case by MTHFR C677T polymorphism, prevails especially under insufficient folate, vitamin B6, and/or vitamin B12 levels [24]. Increased Hcy levels, as a consequence of insufficient remethylation and/ or transsulfuration, have been described to increase the production of reactive oxygen species via autoxidation of homocysteine [25]. In addition, homocysteine and the oxidized metabolite homocysteic acid are described as potent neurotoxins because of its property to be an endogenous NMDA ( $N$-methyl-D-aspartate) receptor agonists [6]. High levels of homocysteine also are associated with DNA damage because the amino acid impairs the DNA repair, supporting neuronal cell death [5]. These processes increase indirectly the vulnerability of neurons to get damaged by $A \beta[26,27]$. There is, moreover, also a direct effect of increased Hcy concentrations on $A \beta$ toxicity, which is due to the enhanced aggregation propensity of $\mathrm{A} \beta_{1-42}$ via the homocysteinylation of lysine residues leading to stabilized soluble oligomeric intermediates [28].

Nutritional factors able to decrease Hcy levels and to cause slowing of cognitive decline and of atrophy in critical brain regions, consistent with modification of the $\mathrm{AD}$ process at least in high risk subjects with baseline vitamin B status, are folate, vitamin B6, and vitamin B12 [4]. Whereas vitamin B6 could increase the synthesis of cystathionine from Hcy and serine by CBS, folate as cofactor for MTHFR and B12 for methionine-synthase might increase under those conditions remethylation of Hcy, betaine could serve also as methyl group donor for 
a

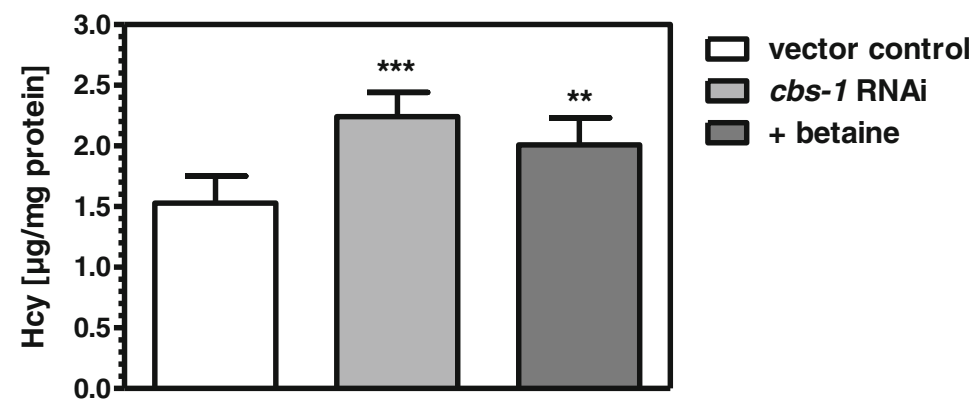

b

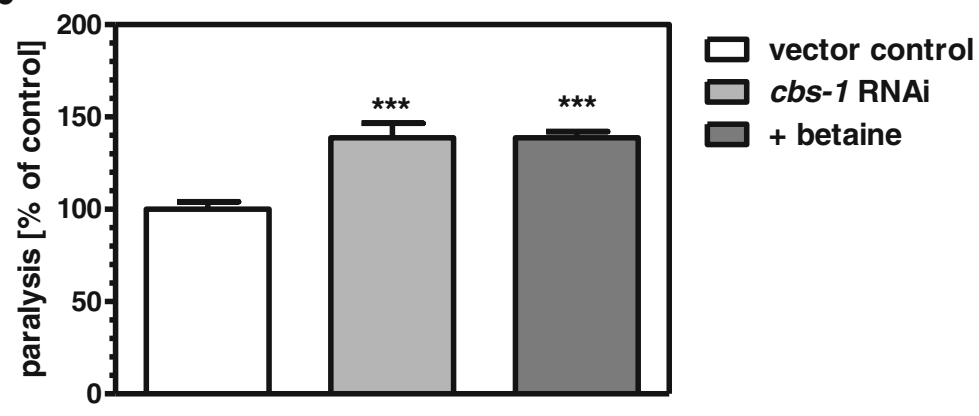

C

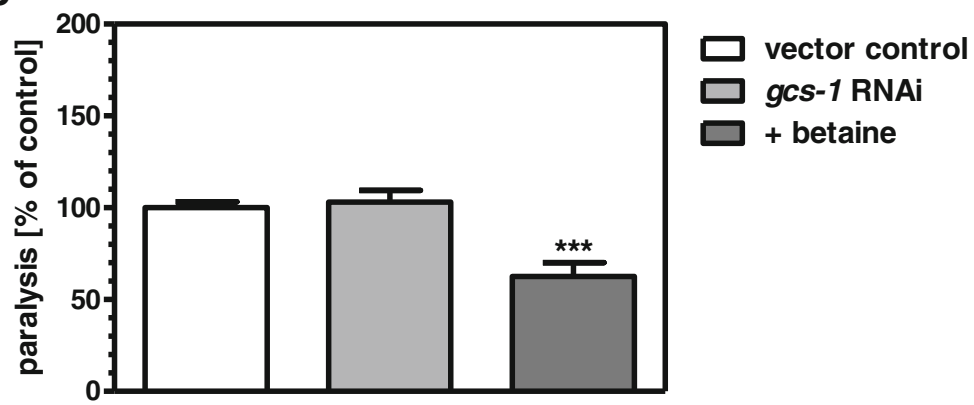

Fig. 3 The glutathione synthesis is not involved in the paralysis reducing effects of betaine. Under cbs-1 RNAi, betaine was no longer able to reduce Hcy level significantly (a) nor to diminish paralysis (b). Knockdown of gcs-1, encoding the key enzyme for glutathione synthesis, did neither affect paralysis nor prevent the paralysis reducing effects of betaine $(\mathbf{c}) .{ }^{* *} p<0.01,{ }^{* * *} p<0.001$ versus vector control

Hcy as mediated by BHMT. Indeed betaine has been shown in this context to arrest Hcy-induced AD-like pathological changes and memory deficits more efficient than supplementation of folate [11]. In C. elegans, however, BHMT is not present due to genetic loss [12]. Accordingly, C. elegans represents the perfect model to investigate whether there are effects of betaine on AD-like phenotypes which are independent on the remethylation of Hcy.

In C. elegans CL2006, expressing a transgene for human $A \beta$, inhibition of remethylation by metr-1 RNA, or transsulfuration by $c b s-1$ RNAi both increased Hcy levels and paralysis. This demonstrates that one-carbon metabolism is functional also in C. elegans as has been observed previously with regard to adaptive responses to dietary restriction [29]. Moreover, the results suggest that Hcy levels are directly associated with the paralytic phenotype in CL2006. The latter was substantiated by the findings that increased Hcy levels by addition of exogenous Hcy lead to increased paralysis whereas lowering of Hcy levels in the presence or absence of exogenous Hcy reduces the paralysis rate to a similar extent at the same dose. Although it might be concluded that lowering of Hcy levels by betaine could be due to activation of MTHFR and methionine-synthase. We did not perceive it as rationale to follow this possibility since enhanced levels of SAM would allosterically activate CBS [30]. We therefore focused on CBS as a target for the observed betaine effects. Indeed betaine was no longer able to reduce Hcy levels or paralysis when increased by $c b s-1$ RNAi. We finally postulated that increased Hcy detoxification under betaine exposure by 
increased cystathionine synthesis could, through cleavage by cystathionase, deliver more cysteine for glutathione synthesis. As described above, enhanced generation of reactive oxygen species is a typical phenomenon of $\mathrm{AD}$ and has been attributed to decreased levels of the brain antioxidant, glutathione [31]. Moreover, lowered cortical glutathione levels were found as a biomarker of early Alzheimer disease pathogenesis [32]. However, in CL2006, the knockdown of $g c s-1$, encoding the ratelimiting enzyme for glutathione synthesis, did neither affect the paralysis rate nor did it prevent betaine from being active in diminishing paralysis.

\section{Conclusions}

In conclusion, our study shows that betaine reduces the A $\beta$-induced degeneration in an Alzheimer model of the nematode $C$. elegans by lowering the Hcy levels. CBS was, moreover, identified as the target enzyme through which betaine exerts its degeneration preventing effects.

\section{Abbreviations}

AD: Alzheimer's disease; A $\beta$ : $\beta$-Amyloid; BHMT: Betaine-homocysteinemethyltransferase; CBS: Cystathionine- $\beta$-synthase; GCS: $\gamma$-Glutamyl-cysteinesynthetase; Hcy: Homocysteine; HRP: Horseradish peroxidase; METR: 5Methyltetrahydrofolate-homocysteine-methyltransferase; MTHFR: 5,10Methylene-tetrahydrofolate-reductase; SAM: S-Adenosylmethionine

\section{Acknowledgements}

Some strains were provided by the CGC, which is funded by NIH Office of Research Infrastructure Programs (P40 OD010440).

\section{Availability of data and materials}

The datasets used and/or analyzed during the current study are available from the corresponding author on reasonable request.

\section{Authors' contributions \\ AL made substantial contributions to acquisition, analysis, and interpretation of data. BD contributed to data acquisition. AL drafted the manuscript. UW made substantial contributions to conception and design as well as interpretation of data and manuscript revision. EF contributed to the data analysis and interpretation as well as manuscript revision. All authors read and approved the final manuscript.}

\section{Ethics approval and consent to participate}

Not applicable

\section{Consent for publication}

Not applicable

\section{Competing interests}

The authors declare that they have no competing interests.

\section{Publisher's Note}

Springer Nature remains neutral with regard to jurisdictional claims in published maps and institutional affiliations.

Received: 17 April 2018 Accepted: 11 July 2018

Published online: 27 July 2018

\section{References}

1. LaFerla FM, Green KN, Oddo S. Intracellular amyloid-beta in Alzheimer's disease. Nat Rev Neurosci. 2007;8(7):499-509.

2. Glenner GG, Wong CW. Alzheimer's disease. Biochem Biophys Res Commun. 2012;425(3):534-9.
3. Selkoe DJ. Alzheimer's disease. Cold Spring Harb Perspect Biol. 2011;3(7): a004577

4. Smith AD, Refsum H. Homocysteine, B vitamins, and cognitive impairment. Annu Rev Nutr. 2016;36:211-39.

5. Vanzin CS, Manfredini V, Marinho AE, et al. Homocysteine contribution to DNA damage in cystathionine $\beta$-synthase-deficient patients. Gene. 2014; 539(2):270-4.

6. Sibarov DA, Abushik PA, Giniatullin R, Antonov SM. GluN2A subunitcontaining NMDA receptors are the preferential neuronal targets of homocysteine. Front Cell Neurosci. 2016;10:246.

7. Lin H-C, Hsieh H-M, Chen Y-H, Hu M-L. S-Adenosylhomocysteine increases beta-amyloid formation in BV-2 microglial cells by increased expressions of beta-amyloid precursor protein and presenilin 1 and by hypomethylation of these gene promoters. Neurotoxicology. 2009;30(4):622-7.

8. Masud R, Baqai HZ. The communal relation of MTHFR, MTR, ACE gene polymorphisms and hyperhomocysteinemia as conceivable risk of coronary artery disease. Appl Physiol Nutr Metab. 2017;42(10):1009-14.

9. Liew SC, Gupta ED. Methylenetetrahydrofolate reductase (MTHFR) C677T polymorphism: epidemiology, metabolism and the associated diseases. Eur J Med Genet. 2015;58(1):1-10.

10. McBreairty LE, Robinson JL, Harding SV, et al. Betaine is as effective as folate at re-synthesizing methionine for protein synthesis during moderate methionine deficiency in piglets. Eur J Nutr. 2016;55(8):2423-30.

11. Chai GS, Jiang X, Ni ZF, et al. Betaine attenuates Alzheimer-like pathological changes and memory deficits induced by homocysteine. J Neurochem. 2013;124(3):388-96.

12. Bito T, Watanabe F. Biochemistry, function, and deficiency of vitamin B12 in Caenorhabditis elegans. Exp Biol Med. 2016;241(15):1663-8.

13. Link CD. Expression of human beta-amyloid peptide in transgenic Caenorhabditis elegans. Proc Natl Acad Sci U S A. 1995;92(20):9368-72.

14. Brenner S. The genetics of Caenorhabditis elegans. Genetics Dev. 1974;77(1): 71-94.

15. Stiernagle T. Maintenance of $C$. elegans, WormBook the online review of $C$. elegans biology, ed. The C. elegans Research Community. 2006:1-11. http:// www.wormbook.org.

16. Lehner B, Tischler J, Fraser AG. RNAi screens in Caenorhabditis elegans in a 96-well liquid format and their application to the systematic identification of genetic interactions. Nat Protoc. 2006;1 (3):1617-20.

17. Timmons $L$, Court $D L$, Fire A. Ingestion of bacterially expressed dsRNAs can produce specific and potent genetic interference in Caenorhabditis elegans. Gene. 2001;263(1-2):103-12.

18. Link C, Taft A, Kapulkin V, et al. Gene expression analysis in a transgenic Caenorhabditis elegans Alzheimer's disease model. Neurobiol Aging. 2003; 24(3):397-413.

19. Haass C, Selkoe DJ. Soluble protein oligomers in neurodegeneration: lessons from the Alzheimer's amyloid beta-peptide. Nat Rev Mol Cell Biol. 2007;8(2):101-12.

20. Tamagno E, Bardini P, Guglielmotto M, et al. The various aggregation states of beta-amyloid 1-42 mediate different effects on oxidative stress, neurodegeneration, and BACE-1 expression. Free Radic Biol Med. 2006; 41(2):202-12.

21. Lin M, Beal MF. Mitochondrial dysfunction and neurodegenerative diseases. Biochim Biophys Acta. 1998;1366(1-2):211-23.

22. Demuro A, Mina E, Kayed R, et al. Calcium dysregulation and membrane disruption as a ubiquitous neurotoxic mechanism of soluble amyloid oligomers. J Biol Chem. 2005;280(17):17294-300.

23. Guglielmotto M, Monteleone D, Piras A, et al. AB1-42 monomers or oligomers have different effects on autophagy and apoptosis. Autophagy. 2014;10(10):1827-43.

24. Troesch $B$, Weber P, Mohajeri MH. Potential links between impaired onecarbon metabolism due to polymorphisms, inadequate B-vitamin status, and the development of Alzheimer's disease. Nutrients. 2016;8(12)

25. Zou CG, Banerjee R. Homocysteine and redox signaling. Antioxid Redox Signal. 2005;7(5-6):547-59.

26. Ho Pl, Collins SC, Dhitavat $\mathrm{S}$, et al. Homocysteine potentiates betaamyloid neurotoxicity: role of oxidative stress. J Neurochem. 2001;78(2): $249-53$.

27. Kruman II, Kumaravel TS, Lohani A, et al. Folic acid deficiency and homocysteine impair DNA repair in hippocampal neurons and sensitize them to amyloid toxicity in experimental models of Alzheimer's disease. J Neurosci. 2002;22(5):1752-62. 
28. Khodadadi S, Riazi GH, Ahmadian S, et al. Effect of N-homocysteinylation on physicochemical and cytotoxic properties of amyloid $\beta$-peptide. FEBS Lett. 2012;586(2):127-31.

29. Klapper M, Findeis D, Koefeler H, Döring F. Methyl group donors abrogate adaptive responses to dietary restriction in C. Elegans. Genes Nutr. 2016;11:4.

30. Ereño-Orbea J, Majtan T, Oyenarte I, et al. Structural insight into the molecular mechanism of allosteric activation of human cystathionine $\beta$ synthase by S-adenosylmethionine. Proc Natl Acad Sci U S A. 2014;111(37) E3845-52.

31. Saharan S, Mandal PK. The emerging role of glutathione in Alzheimer's disease. J Alzheimers Dis. 2014;40(3):519-29.

32. Chiang GC, Mao X, Kang G, et al. Relationships among cortical glutathione levels, brain amyloidosis, and memory in healthy older adults investigated in vivo with $1 \mathrm{H}-\mathrm{MRS}$ and Pittsburgh compound-B PET. AJNR Am J Neuroradiol. 2017;38(6):1130-7.

Ready to submit your research? Choose BMC and benefit from:

- fast, convenient online submission

- thorough peer review by experienced researchers in your field

- rapid publication on acceptance

- support for research data, including large and complex data types

- gold Open Access which fosters wider collaboration and increased citations

- maximum visibility for your research: over $100 \mathrm{M}$ website views per year

At BMC, research is always in progress.

Learn more biomedcentral.com/submissions 